PHYSICAL REVIEW A 83, 052113 (2011)

\title{
Fourth moments reveal the negativity of the Wigner function
}

\author{
Adam Bednorz ${ }^{1, *}$ and Wolfgang Belzig ${ }^{2}$ \\ ${ }^{1}$ Faculty of Physics, University of Warsaw, Hoża 69, PL-00681 Warsaw, Poland \\ ${ }^{2}$ Fachbereich Physik, Universität Konstanz, D-78457 Konstanz, Germany
}

(Received 7 March 2011; published 12 May 2011)

\begin{abstract}
The presence of unique quantum correlations is the core of quantum-information processing and general quantum theory. We address the fundamental question of how quantum correlations of a generic quantum system can be probed using correlation functions defined for quasiprobability distributions. In particular, we discuss the possibility of probing the negativity of a quasiprobability by comparing moments of the Wigner function. We show that one must take at least the fourth moments to find the negativity in general and the eighth moments for states with a rotationally invariant Wigner function.
\end{abstract}

DOI: 10.1103/PhysRevA.83.052113

PACS number(s): 03.65.Ta, 42.50.Xa

\section{INTRODUCTION}

The von Neumann projective detection scheme [1] allows one to measure simultaneously only commuting quantum observables. It is impossible to define a useful probability of measurement outcomes for noncommuting observables, like position and momentum, consistent with a projective measurement. To circumvent this problem, it is possible to define a quasiprobability — the Wigner function [2] — consistent with the projective measurement but taking sometimes negative values. Because of the Heisenberg uncertainty principle, the Wigner function cannot be directly measurable in the von Neumann sense. However, one can find it by indirect measurements like the homodyne tomography [3], assuming a special detection scheme, and using the inverse radon or Abel transformations. In this scheme, it is essential to use efficient photon counters. The negativity of the Wigner function, with the position and momentum replaced by the electric and magnetic field, has been demonstrated using single photons and other states $[4,5]$.

Recently, it has become possible to measure the moments of the microwave electromagnetic field $[6,7]$. The microwave frequencies make it difficult to build efficient photon counters although there exists a proposal [8]. Using instead linear amplifiers leads to a large detection noise and, contrary to the homodyne detection, one cannot reconstruct the complete Wigner function. Hence it is relevant to ask if we can show the negativity of the Wigner function having access only to correlations of position and momentum.

The detection schemes based on linear amplifiers are easier to interpret in terms of the quantum weak measurement [9] than in the traditional projective scheme. The interpretation of weak measurements leads to paradoxes similar to the negative Wigner function. In the weak measurement picture, the detector just adds a large white noise of the outcome. The detection noise contains no information about the measured system and can be subtracted by a deconvolution of the resulting probability distribution. However, the remaining distribution is a quasiprobability, taking sometimes negative values. In the case of momentum and position the quasiprobability corresponds to the Wigner function [10-12]. The

\footnotetext{
*Adam.Bednorz@fuw.edu.pl
}

deconvolution is in principle experimentally feasible, but since usually one has access only to correlation functions, it is useful to develop schemes for them to detect quantum correlations. Generally, we have shown previously that the knowledge of a finite number of moments of a quasiprobability can show its negativity [12].

In this paper, we discuss how the negativity of the Wigner function can appear as a violation of an inequality involving only finite moments. We emphasize that there exist examples violating the Cauchy-Bunyakovsky-Schwarz inequality by fourth or higher moments for position and momentum (or second moments for their squares or intensities) $[13,14]$ but only using the Glauber-Sudarshan $P$ function [15], whose negativity is much easier to demonstrate, compared to Wigner function. We will show in the following that it is possible to prove the negativity of the Wigner function using only the fourth moments for position and momentum although the violating state is complicated. For rotationally invariant states, mixtures of Fock states for a harmonic Hamiltonian with a rotationally invariant Wigner function, we show that one needs at least the eighth moment to show the negativity of quasiprobability. Additionally, we provide a connection between the continuous time-resolved measurement of position and momentum, which permits one to find moments of a single-time Wigner function by deconvolution in a harmonic system.

The paper is organized as follows. We start from recalling basic properties of the Wigner function, including its negativity. Next, we demonstrate violation of classical inequalities by fourth moment for a special state and eighth moments for a rotationally invariant state. Finally, we present a way to find moments in continuous measurement by deconvolution and close with conclusions.

\section{WIGNER FUNCTION}

We begin with the standard construction of the Wigner function. Let us define the dimensionless Hermitian position and momentum operators, $\hat{x}, \hat{p}$ with the property $\hat{x} \hat{p}-\hat{p} \hat{x}=$ $[\hat{x}, \hat{p}]=i$ ( $\hbar$ is incorporated in the definition of $x$ and $p$ ). One can always make a rescaling $\hat{x} \rightarrow \lambda_{x} \hat{x}, \hat{p} \rightarrow \lambda_{p} \hat{p}$ later to recover the proper dimension. Instead of $x$ and $p$, in optical 
systems one can use the electric and magnetic fields $E$ and $B$ for a given mode. The Wigner function is defined as [2]

$$
W(x, p)=\int \frac{d \chi d \xi}{(2 \pi)^{2}} e^{-i(\chi x+\xi p)} \operatorname{Tr} e^{i(\chi \hat{x}+\xi \hat{p})} \hat{\rho} .
$$

Here $\hat{\rho}$ is the density matrix of the system. One essential property of the Wigner function is that its marginals correspond to the standard projective probability,

$$
\begin{aligned}
& \rho(x)=\operatorname{Tr} \hat{\rho} \delta(x-\hat{x})=\int W(x, p) d p, \\
& \rho(p)=\operatorname{Tr} \hat{\rho} \delta(p-\hat{p})=\int W(x, p) d x .
\end{aligned}
$$

Similar relations can be found for arbitrary linear combinations of $x$ and $p$ used, e.g., to experimentally extract the Wigner function [4]. We stress that, contrary to the $P$ function, the Wigner function definition does not involve any characteristic harmonic frequency $\omega$. Nevertheless, it is convenient to represent $\hat{\rho}$ in terms of coherent or Fock states defined for a harmonic oscillator.

The Wigner function can never be measured directly, which follows from the Heisenberg uncertainty principle and the consistency of quantum measurements. It cannot be measurable as a probability because it takes negative values. To show this, we introduce recursively the Fock basis states $|n+1\rangle=\hat{a}^{\dagger}|n\rangle / \sqrt{n+1}$ for $n=0,1,2, \ldots$ and $\langle 0 \mid 0\rangle=1$, $\hat{a}|0\rangle=0$. They form the complete set of eigenstates of the Hamiltonian,

$$
\hat{H}=\hbar \omega \hat{a}^{\dagger} \hat{a}, \quad \hat{a}=(\hat{x}+i \hat{p}) / \sqrt{2},
$$

with the property $\left[\hat{a}, \hat{a}^{\dagger}\right]=\hat{1}$. In this notation, we have set $m=\omega^{-1}$. We have $\hat{H}|n\rangle=\hbar \omega n|n\rangle$ and

$$
W(x, p)=e^{-\left(x^{2}+p^{2}\right)}\left[2\left(x^{2}+p^{2}\right)-1\right] / \pi,
$$

with $W(0,0)=-1 / \pi<0$ for $\hat{\rho}=|1\rangle\langle 1|$. In general, for pure states $\hat{\rho}=|\psi\rangle\langle\psi|$, the Wigner function is always somewhere negative unless the state has a Gaussian wave function [16]. The directly measurable quantity is $W[x(t)]$, which allows one to retrieve the full Wigner function by a numerically difficult inverse radon transform (or Abel transform if the state can be assumed to be rotationally invariant) [3]. The statistics needs a lot of repetitions of the experiment. Moreover, it needs a strong, projective measurement, excluding any external noise. This condition is possible to fulfill theoretically but the experimental implementation relies much on a hardly verifiable detection scheme (based on efficient photon counters). More common is the situation with some, in many cases even large, detection noise, which, on the other hand, can be well estimated and subtracted [6], as we show in Sec. V. This latter method makes it impossible, however, to find the whole Wigner function. In this scheme, one can only find a finite number of moments of the Wigner function. We stress that the moments of the Wigner function are naturally measured in a continuous weak measurement (and not the $P$ and $Q$ function). Furthermore, as we show in Sec. V by a suitable processing of the continuous signal of position only, all moments involving momentum can be extracted as well.

Motivated by the continuous measuring scheme, it is natural to pose the following questions. Can we say something about the negativity of the Wigner function from the knowledge of a finite number of its moments? What is the smallest order of the moment to show that the Wigner function is negative? How complicated must the violating state be? Below we answer all these questions.

\section{NONCLASSICAL MOMENTS: GENERAL CASE}

An arbitrary probability distribution must satisfy the generic inequality:

$$
\left\langle f^{2}(x, p)\right\rangle \geqslant 0 \text {. }
$$

Once the above inequality is violated, the candidate for a probability must be somewhere negative and fails the test to be a real, positive probability. In the case of the Wigner function, we consider the following expansion:

$$
\begin{aligned}
f(x, p)= & \sum_{n m} c_{n m} x^{n} p^{m}=c_{00}+c_{10} x+c_{01} p \\
& +c_{20} x^{2}+c_{11} x p+c_{02} p^{2}+c_{30} x^{3}+\cdots,
\end{aligned}
$$

with real $c_{n m}$ for $n, m \geqslant 0$. We say that $f$ is of the order $k$ if there exists a nonzero $c_{n m}$ for $n+m=k$ and all $c_{n m}=0$ for $n+m>k$.

We focus on the following task: find the state $\hat{\rho}$ that violates

$$
\left\langle f^{2}(x, p)\right\rangle_{W} \geqslant 0
$$

for the $f$ of the lowest possible order. One can easily show that $k>1$. If $f(x, p)=c_{0}+c_{10} x+c_{01} p$, then

$$
\left\langle f^{2}(x, p)\right\rangle_{W}=\operatorname{Tr} f^{2}(\hat{x}, \hat{p}) \hat{\rho} \geqslant 0
$$

Next, the case $k=2$ can be reduced by linear transformations on the phase space, namely rotating $(x, p) \rightarrow(x \cos \phi+$ $p \sin \phi, p \cos \phi-x \sin \phi)$, squeezing $(x, p) \rightarrow\left(g x, g^{-1} p\right)$ and shifting $(x, p) \rightarrow\left(x+x_{0}, p+p_{0}\right)$, which is justified in both the classical and the quantum case to $f_{a}(x, p)=x^{2}+$ $p^{2}+c_{0}$ or $f_{b}(x, p)=2 x p+c_{0}$. Other cases either reduce to $f_{a, b}$ or will never violate the inequality (7). It is enough to consider pure states $\hat{\rho}=|\psi\rangle\langle\psi|$ because violating a mixed state must contain a violating pure state in its decomposition. Remember that choosing the Fock space of the harmonic oscillator we do not lose generality, since an arbitrary state can be written in terms of Fock states even if the Hamiltonian is different from Eq. (3).

For $f_{a}$, one can show that $x^{2}+p^{2}=2 a^{*} a$ and

$$
\begin{gathered}
\left\langle 2 a^{*} a\right\rangle_{W}=\operatorname{Tr}(2 \hat{n}+1) \hat{\rho}, \\
\left\langle\left(2 a^{*} a\right)^{2}\right\rangle_{W}=\operatorname{Tr}\left(4 \hat{n}^{2}+4 \hat{n}+2\right) \hat{\rho},
\end{gathered}
$$

with $\hat{n}=\hat{a}^{\dagger} \hat{a}$. Hence we can consider only the $|n\rangle$ states but $4 n^{2}+4 n+2>(2 n+1)^{2}$ so $f_{a}$ will never violate Eq. (7).

We have $2 x p=i\left(a^{* 2}-a^{2}\right)$ and

$$
\begin{gathered}
\left\langle\left(a^{* 2}-a^{2}\right)\right\rangle_{W}=\operatorname{Tr}\left(\hat{a}^{\dagger 2}-\hat{a}^{2}\right) \hat{\rho}, \\
\left\langle\left(a^{* 2}-a^{2}\right)^{2}\right\rangle_{W}=\operatorname{Tr}\left[\left(\hat{a}^{4}+\hat{a}^{\dagger 4}\right) \hat{\rho}\right]-2\left\langle\left(a^{*} a\right)^{2}\right\rangle_{W} .
\end{gathered}
$$

This gives also the bound $\left\langle f_{b}^{2}\right\rangle_{W} \geqslant-1$ following from

$$
\left\langle f_{b}^{2}\right\rangle_{W}+1=\operatorname{Tr} \hat{\rho}\left[i\left(\hat{a}^{\dagger 2}-\hat{a}^{2}\right)+c_{0}\right]^{2} \geqslant 0 .
$$


We can consider separately $|\psi\rangle$ made of odd states $|2 k+1\rangle$ and of even states $|2 k\rangle$ because $f_{b}$ preserves parity, since operators appear pairwise.

The search for the violation of Eq. (7) reduces to the diagonalization of a quadratic form, parametrized by $c_{0}$, which is easily accomplished numerically. The outcome of the search is that we need at least five states of Fock space and the largest violation of Eq. (7) occurs for $c_{0}=0$. The simplest state violating Eq. (7) with $f_{b}$ has the structure

$$
|\psi\rangle=v_{0}|0\rangle+v_{4}|4\rangle+v_{8}|8\rangle+v_{12}|12\rangle+v_{16}|16\rangle .
$$

Since $c_{0}=0$ and $x p$ shifts the state number by 2, we omit states $|4 k+2\rangle$. The coefficients $\left(v_{0}, v_{4}, v_{8}, v_{12}, v_{16}\right)$ form the eigenvector of the matrix,

$$
\left(\begin{array}{ccccc}
A_{0} & B_{1} & 0 & 0 & 0 \\
B_{1} & A_{1} & B_{2} & 0 & 0 \\
0 & B_{2} & A_{2} & B_{3} & 0 \\
0 & 0 & B_{3} & A_{3} & B_{4} \\
0 & 0 & 0 & B_{4} & A_{4}
\end{array}\right),
$$

with the smallest eigenvalue for

$$
\begin{gathered}
A_{k}=32 k^{2}+8 k+1, \\
B_{k}=-\sqrt{4 k(4 k-1)(4 k-2)(4 k-3)} .
\end{gathered}
$$

The fact that the matrix has a negative eigenvalue follows from the negativity of its determinant, equal to -10447775 . The numerical diagonalization gives $\lambda_{\min }=\left\langle(2 x p)^{2}\right\rangle_{W}=-0.036$ and its normalized eigenvector is given by $\left(v_{0}, v_{4}, v_{8}, v_{12}, v_{16}\right)=$ $(0.973,0.206,0.0897,0.042,0.0161)$. Going to more complicated states allows for an even stronger violation. However, we stress that our numerical search has shown that at least a superposition of five Fock states is necessary. Hence we draw the important conclusion that showing the nonclassicality of the Wigner function using only moments requires at least fourth moments and states involving coherent superposition of at least five states.

The violation for a general state is bounded by Eq. (11). It is interesting to note that one can find a state with $\left\langle f_{b}^{2}\right\rangle_{W}$ arbitrarily close to -1 . We can conveniently describe such a state by its wave function in position space $|\psi\rangle=\int d x \psi(x)|x\rangle$, with $\langle x \mid y\rangle=\delta(x-y), \hat{x}=x$, and $\hat{p}=-i d / d x$. We write Eq. (11) in the form

$$
\left\langle f_{b}^{2}\right\rangle_{W}+1=\int|\phi(x)|^{2} d x
$$

and

$$
\phi(x)=\left(\hat{x} \hat{p}+\hat{p} \hat{x}+c_{0}\right) \psi(x)=\left(c_{0}-i-2 i x d / d x\right) \psi(x) .
$$

To get the minimum of $\left\langle f_{b}^{2}\right\rangle_{W}$, we should make $\phi(x)$ as small as possible. However, trying to get $\left\langle f_{b}^{2}\right\rangle_{W}=-1$ implies $\phi=0$ and $\psi(x) \sim|x|^{-\left(1+i c_{0}\right) / 2}$, which cannot be normalized. Instead, we take a regularized wave function

$$
\psi(x)=e^{-|x| / 2}|x|^{\epsilon-1 / 2} / \sqrt{2 \Gamma(2 \epsilon)},
$$

with $\epsilon>0$ and the $\Gamma$ function $\Gamma(z)$.
The Wigner function reads

$W(x, p)=\frac{|x / 2|^{2 \epsilon}}{\pi \Gamma(\epsilon)}\left[\frac{\pi J_{\epsilon}(2 x p)}{|x p|^{\epsilon} e^{|x|}}+\operatorname{Re} \frac{2 K_{\epsilon}(|x|+2 i x p)}{(i p x+|x| / 2)^{\epsilon}}\right]$

with $W \sim J_{0}(2 x p)-Y_{0}(2 x p)$ for $\epsilon \sim 0$ and $J, K, Y$ denoting Bessel functions. We get $\phi(x)=-i(2 \epsilon-|x|) \psi(x)$, which gives $\left\langle(2 x p)^{2}\right\rangle_{W}=2 \epsilon-1$. This result shows that we can get arbitrarily close to the limiting value -1 with $\epsilon \rightarrow 0$; however, the case $\epsilon=0$ is impossible as the wave function would not be normalizable. We stress that Eq. (17) represents only an example of a state with $\left\langle f_{b}^{2}\right\rangle_{W} \rightarrow-1$ and one can certainly find such states also in the family of analytic functions, e.g., a sequence of states in finite Fock spaces.

\section{NONCLASSICAL MOMENTS AT ROTATIONAL INVARIANCE}

We ask the further question: what is the lowest order of $f$ that violates Eq. (7) for rotationally invariant states (considering the Wigner function in phase space)? The rotational invariance is intimately connected with the harmonic dynamics (3), which induces a rotation frequency $\omega$. It is important since it is often difficult to control experimentally the phase of the photonic state, corresponding to the polar angle in the phase space. If the phase is smeared out, then the state is rotationally invariant, described by a mixture of Fock states $|n\rangle$, namely $\hat{\rho}=\sum_{n} p_{n}|n\rangle\langle n|$. Again, it is enough to focus on pure Fock states. Next, the parts of $f$ with different parity with respect to $x$ or $p$ can be considered separately as their cross products integrate out to zero for Fock states. The function $f_{b}$ reduces to $f_{a}$ for Fock states so it cannot violate Eq. (7). The only nontrivial case with $k=3$ is then

$$
f_{c}(x, p)=x\left(x^{2}+p^{2}\right)+\left(c_{30}-1\right) x^{3}+c_{10} x .
$$

One can find that for a rotationally invariant distribution

$$
\begin{aligned}
\left\langle f_{c}^{2}\right\rangle= & \left(5 c_{30}^{2}+2 c_{30}+1\right)\left\langle r^{6}\right\rangle / 16 \\
& +\left(3 c_{30}+1\right) c_{10}\left\langle r^{4}\right\rangle / 4+c_{10}^{2}\left\langle r^{2}\right\rangle / 2,
\end{aligned}
$$

where $r^{2}=x^{2}+p^{2}$. The straightforward analysis of the above quadratic form gives its minimum located at

$$
\begin{gathered}
c_{30}=\frac{3\left\langle r^{4}\right\rangle^{2}-2\left\langle r^{6}\right\rangle\left\langle r^{2}\right\rangle}{10\left\langle r^{6}\right\rangle\left\langle r^{2}\right\rangle-9\left\langle r^{4}\right\rangle^{2}}, \quad c_{10}=\frac{-\left\langle r^{4}\right\rangle\left\langle r^{6}\right\rangle}{10\left\langle r^{6}\right\rangle\left\langle r^{2}\right\rangle-9\left\langle r^{4}\right\rangle^{2}}, \\
\left\langle f_{c}^{2}\right\rangle=\frac{\left\langle r^{6}\right\rangle\left(\left\langle r^{6}\right\rangle\left\langle r^{2}\right\rangle-\left\langle r^{4}\right\rangle^{2}\right)}{2\left(10\left\langle r^{6}\right\rangle\left\langle r^{2}\right\rangle-9\left\langle r^{4}\right\rangle^{2}\right)} .
\end{gathered}
$$

On the one hand, for the Fock state $|n\rangle$ one finds $\left\langle r^{2}\right\rangle_{W}=$ $2 n+1,\left\langle r^{4}\right\rangle_{W}=4 n^{2}+4 n+2$, and $\left\langle r^{6}\right\rangle_{W}=8 n^{3}+12 n^{2}+$ $16 n+6$, so $\left\langle r^{6}\right\rangle_{W}\left\langle r^{2}\right\rangle_{W}-\left\langle r^{4}\right\rangle_{W}^{2}=12 n^{2}+12 n+2>0$. Hence $\left\langle f_{c}^{2}\right\rangle$ will never be negative for Fock states.

The negativity appears for $k=4$, namely for $f_{d}(x, p)=$ $r^{4}+c_{20} r^{2}+c_{0}$, taking the state $|1\rangle$. One gets

$$
\left\langle f_{d}^{2}\right\rangle_{W}=10 c_{20}^{2}+c_{0}^{2}+6 c_{20} c_{0}+84 c_{20}+20 c_{0}+216 .
$$

The minimum of the above function is located at $c_{20}=-12$ and $c_{0}=26$ and equals $\left\langle f_{d}^{2}\right\rangle_{W}=-28$. This is our second result: we need the eighth moments and not less to show the nonclassicality of rotationally invariant states. 


\section{MOMENTS FROM CONTINUOUS MEASUREMENT}

The concept of the Wigner function is very general as it only relies of the fact that two observables do not commute. However, in many real experiments, the observables are measured continuously and, hence, it is necessary to extend the concept of a quasiprobability to continuous time-resolved measurements. To this end, we define a time-extended Wigner functional, in accord with general considerations [10-12], by a path integral

$$
\mathcal{W}[x, p]=\int D \chi D \xi e^{S[\chi, \xi]-\int i d t[\chi(t) x(t)+\xi(t) p(t)]},
$$

$e^{S[\chi, \xi]}=\operatorname{Tr} \mathcal{T} e^{\int i d t[\chi(t) \hat{x}(t)+\xi(t) \hat{p}(t)] / 2} \hat{\rho} \tilde{\mathcal{T}} e^{\int i d t[\chi(t) \hat{x}(t)+\xi(t) \hat{p}(t)] / 2}$,

where $\mathcal{T}(\tilde{\mathcal{T}})$ denotes (anti)time ordering, $\hat{A}(t)$ denotes an operator in the Heisenberg picture, and $\hat{\rho}$ is the initial density matrix. Such a functional can be measured as a convolution with some detection noise [12],

$$
\rho[x, p]=\int D x^{\prime} D p^{\prime} \rho_{d}\left[x-x^{\prime}, p-p^{\prime}\right] \mathcal{W}\left[x^{\prime}, p^{\prime}\right],
$$

where $\rho_{d}$ corresponds to the large detection white noise, independent of the state of the system. This corresponds to the experimental detection scheme, in which moments of $\mathcal{W}$ can be found by deconvolution $[6,17]$. The measurable quantities are the cumulants

$$
\left\langle\left\langle A_{1} \cdots A_{n}\right\rangle\right\rangle=\left.(-i)^{n} \frac{\partial^{n} S\left(\chi_{1}, \ldots, \chi_{n}\right)}{\partial \chi_{1} \cdots \partial \chi_{n}}\right|_{\chi=0}
$$

and the moments

$$
\left\langle A_{1} \cdots A_{n}\right\rangle=\left.(-i)^{n} \frac{\partial^{n} e^{S\left(\chi_{1}, \ldots, \chi_{n}\right)}}{\partial \chi_{1} \cdots \partial \chi_{n}}\right|_{\chi=0},
$$

where the cumulant-generating function (CGF) is defined

$$
S\left(\chi_{1}, \ldots, \chi_{n}\right)=\ln \left\langle e^{i\left(A_{1} \chi_{1}+\cdots+A_{n} \chi_{n}\right)}\right\rangle .
$$

Knowing the cumulants of $\rho$ and $\rho_{d}$, one can find the cumulants and moments of $\mathcal{W}$ due to the relation between CGFs,

$$
S_{\rho}=S_{d}+S_{\mathcal{W}}
$$

Now let us focus on a harmonic oscillator described by the Hamiltonian (3). In the Heisenberg picture, $\hat{a}(t)=e^{-i \omega t} \hat{a}$. Due to the fact that all operators in Eq. (23) commute to $c$ numbers, one can now write

$$
e^{S[\chi, \xi]}=\operatorname{Tr} e^{\int i d t[\chi(t) \hat{x}(t)+\xi(t) \hat{p}(t)]} \hat{\rho} .
$$

One can see also that the Wigner functional (23) vanishes outside classical trajectories, namely

$$
[d a(t) / d t+i \omega a(t)] W[x, p]=0, \quad a=(x+i p) / \sqrt{2} .
$$

It leads to a simple picture: once the initial distribution $W(x, p)$ is known, the general functional $\mathcal{W}[x, p]$ is obtained by evolving along classical trajectories. Therefore, for a harmonic
Hamiltonian, the complete Wigner functional is not necessary for the theoretical calculations as it reduces to the single-time Wigner function. However, the classical evolution is helpful for the experimental detection schemes. It is worth collecting all data from a continuous (weak) monitoring of $x(t)$ and/or $p(t)$ and constructing the initial Wigner function by a reverse mapping of the classical dynamics.

Let us introduce the notation for the marginal distributions:

$$
\begin{gathered}
\mathcal{W}[x]=\int D p \mathcal{W}[x, p], \\
\mathcal{W}\left(x_{1}, \ldots, x_{n}, p_{1}, \ldots, p_{m}\right) \\
=\int D x D p \mathcal{W}[x, p] \delta\left(x_{1}-x\left(t_{1}\right)\right) \cdots \delta\left(x_{n}-x\left(t_{n}\right)\right) \\
\times \delta\left(p_{1}-p\left(t_{1}^{\prime}\right)\right) \cdots \delta\left(p_{m}-p\left(t_{m}^{\prime}\right)\right) .
\end{gathered}
$$

We shall switch the notation $x_{k} \rightarrow x\left(t_{k}\right)$ when it is unambiguous. It is easy to show that

$$
\mathcal{W}[x(0), x(\pi / 2 \omega)]=\mathcal{W}[x(0), p(0)]=W(x, p),
$$

so one can obtain the usual Wigner function also from only position measurement, but at different times, as $p(0)=$ $x(\pi / 2 \omega)$ follows from equation of motion (30). More generally, $W(x, p)$ can be obtained also from the appropriate Fourier components, namely

$$
\begin{aligned}
W(x, p)= & \int D x^{\prime} \delta\left(x-\int_{0}^{t_{0}} 2 \cos (\omega t) x^{\prime}(t) / t_{0}\right) \\
& \times \delta\left(p-\int_{0}^{t_{0}} 2 \sin (\omega t) x^{\prime}(t) / t_{0}\right) \mathcal{W}\left[x^{\prime}\right],
\end{aligned}
$$

where $t_{0} \gg \omega^{-1}$ is the total averaging time. We stress that the averaging time is limited by the backaction of the detector, which protects - by means of the detection noise - the Wigner function from its exact measurement in a single run of an experiment.

\section{CONCLUSION}

We have demonstrated that the negativity of the Wigner function can be shown using only its moments but at least of fourth order. As also shown in other contexts, we confirmed that fourth-order correlations are necessary to show nonclassicality of quantum mechanics [12]. We believe this is fundamentally important for probing nonclassicality by means of weak measurement, when one has access only to moments. We believe that our results will stimulate an effort to measure higher-order correlations in optical cavities by means of linear detectors and amplifiers, even if they add large detection noise.

\section{ACKNOWLEDGMENT}

We acknowledge financial support from the DFG through SFB 767 Controlled Nanosystems and SP 1285 Semiconductor Spintronics.
[1] J. von Neumann, Mathematical Foundations of Quantum Mechanics (Princeton University Press, Princeton, NJ, 1932).

[2] E. P. Wigner, Phys. Rev. 40, 749 (1932); M. Hillery, R. F. O'Connell, M. O. Scully, and E. P. Wigner, Phys. Rep. 106,
121 (1984); W. P. Schleich, Quantum Optics in Phase Space (Wiley-VCH, Weinheim, 2001).

[3] A. I. Lvovsky and M. G. Raymer, Rev. Mod. Phys. 81, 299 (2009). 
[4] A. I. Lvovsky, H. Hansen, T. Aichele, O. Benson, J. Mlynek, and S. Schiller, Phys. Rev. Lett. 87, 050402 (2001).

[5] A. Zavatta, S. Viciani, and M. Bellini, Phys. Rev. A 70, 053821 (2004); A. Ourjoumtsev, R. Tualle-Brouri, and P. Grangier, Phys. Rev. Lett. 96, 213601 (2006).

[6] E. P. Menzel, F. Deppe, M. Mariantoni, M. A. AraqueCaballero, A. Baust, T. Niemczyk, E. Hoffmann, A. Marx, E. Solano, and R. Gross, Phys. Rev. Lett. 105, 100401 (2010).

[7] D. Bozyigit et al., Nat. Phys. 7, 154 (2011); M. P. da Silva et al., Phys. Rev. A 82, 043804 (2010); C. Eichler et al., e-print arXiv:1011.6668.

[8] G. Romero, J. J. Garcia-Ripoll, and E. Solano, Phys. Rev. Lett. 102, 173602 (2009).

[9] Y. Aharonov, D. Z. Albert, and L. Vaidman, Phys. Rev. Lett. 60, 1351 (1988).

[10] M. Tsang, Phys. Rev. A 80, 033840 (2009).
[11] B. Berg, L. I. Plimak, A. Polkovnikov, M. K. Olsen, M. Fleischhauer, and W. P. Schleich, Phys. Rev. A 80, 033624 (2009).

[12] A. Bednorz and W. Belzig, Phys. Rev. Lett. 105, 106803 (2010).

[13] M. D. Reid and D. F. Walls, Phys. Rev. A 34, 1260 (1986).

[14] E. Shchukin, Th. Richter, and W. Vogel, Phys. Rev. A 71, 011802(R) (2005).

[15] E. C. G. Sudarshan, Phys. Rev. Lett. 10, 277 (1963); R. J. Glauber, Phys. Rev. 131, 2766 (1963).

[16] R. L. Hudson, Rep. Math. Phys. 6, 249 (1974); C. Piquet, Comptes rendus hebdomadaires des séances de l'Académie des sciences. Série A, Sciences mathématiques 279, 107 (1974).

[17] W. Vogel, Phys. Rev. Lett. 84, 1849 (2000). 